\section{GW23-e2591 TRANSFORMING GROWTH FACTOR-B2 RELEASED BY MESENCHYMAL STEM CELLS PRECONDITIONED WITH HIGH DENSITY LIPOPROTEIN PROTECTS CARDIOMYOCYTES FROM HYPOXIA}

doi:10.1136/heartjnl-2012-302920a.58

\footnotetext{
${ }^{1}$ Jian-feng Xu, ${ }^{1} J u-y i n g ~ Q i a n,{ }^{2}$ Li Lin, 'Ai-jun Sun, ${ }^{1} Y u n-z e n g ~ Z o u,{ }^{1} J u n-b o ~ G e$,

${ }^{1}$ Juying Oian. 'Department of Cardiology, Zhongshan Hospital, Fudan University, Shanghai 200032, China; ${ }^{2}$ Department of Cardiology, Eastern Hospital, Tongji

University, Shanghai 200120, China
}

Objectives The effect of high density lipoprotein (HDL) on the secretome of mesenchymal stem cells (MSCs) has not been well elucidated yet. The aim of the study is to examine the hypothesis that preconditioning with HDL promotes MSCs secretome, subsequently protecting cardiomyocytes from hypoxia.

Methods MSCs derived from bone marrow of rats were preconditioned with $\mathrm{HDL}$ in various concentrations or for different periods. Afterwards, the mRNA of some soluble factors (including VEGF, bFGF, HGF, TGF- 32 ) in MSCs was analysed by real-time PCR, and the levels of above factors in supernatant were analysed by ELISA. Cardiomyocytes of neonatal rats were exposed to hypoxia for $12 \mathrm{~h}$, incubated in culture medium which were respectively added with the supernatant filtered from MSCs and HDL cultured system (MSCs+HDL), from MSCs alone cultured medium (MSCs), or the same terminal concentration of HDL (HDL) or PBS (Control). Apoptosis of cardiomyocytes was evaluated by TUNEL assay and western-blot. RNA interference was administrated to confirm the effect of soluble factors on the cardiomyocytes (siRNA) apoptosis, in which flow cytometry was addressed to evaluate the apoptosis rate.

Results When MSCs were exposed to HDL, the expression of TGF$\beta 2$, both mRNA level in MSCs and protein level in supernatant, increased significantly in a time- and concentration-dependent manner. Group(MSCs+HDL) exhibited remarkable lower apoptosis rate compared with Group(Control), Group(HDL), Group(MSCs) (TUNEL: $(10.23 \pm 5.68) \%$ vs $(28.36 \pm 4.98) \%$, vs $(16.38 \pm 5.38) \%$, vs $(17.82 \pm 6.24) \%$, p value all $<0.05$; bcl- $2 /$ bax: $(0.846 \pm 0.16)$ vs $(0.156$ $\pm 0.03)$, or $(0.411 \pm 0.10)$, or $(0.346 \pm 0.08)$, $p$ value all $<0.05)$. After knocked down the TGF- $\beta 2$ gene, Group (siRNA) showed a significant higher apoptosis rate compared with Group (MSCs+HDL) $[(10.28 \pm 1.33) \%$ vs $(5.34 \pm 0.67) \%, \mathrm{p}<0.05)$.

Conclusions TGF- $\beta 2$ released by mesenchymal stem cells preconditioned with high density lipoprotein protects cardiomyocytes from injury of hypoxia. 